\title{
A comparison of commercial light-emitting diode baited suction traps for surveillance of Culicoides in northern Europe
}

Andrew Hope ${ }^{1 *}$, Simon Gubbins ${ }^{1}$, Christopher Sanders ${ }^{1}$, Eric Denison ${ }^{1}$, James Barber ${ }^{1}$, Francesca Stubbins $^{1}$, Matthew Baylis ${ }^{2,3}$ and Simon Carpenter ${ }^{1}$

\begin{abstract}
Background: The response of Culicoides biting midges (Diptera: Ceratopogonidae) to artificial light sources has led to the use of light-suction traps in surveillance programmes. Recent integration of light emitting diodes (LED) in traps improves flexibility in trapping through reduced power requirements and also allows the wavelength of light used for trapping to be customized. This study investigates the responses of Culicoides to LED light-suction traps emitting different wavelengths of light to make recommendations for use in surveillance.

Methods: The abundance and diversity of Culicoides collected using commercially available traps fitted with Light Emitting Diode (LED) platforms emitting ultraviolet (UV) (390 nm wavelength), blue (430 nm), green (570 nm), yellow $(590 \mathrm{~nm})$, red $(660 \mathrm{~nm})$ or white light $(425 \mathrm{~nm}-750 \mathrm{~nm}$ with peaks at $450 \mathrm{~nm}$ and $580 \mathrm{~nm})$ were compared. A Centre for Disease Control (CDC) UV light-suction trap was also included within the experimental design which was fitted with a 4 watt UV tube (320-420 nm). Generalised linear models with negative binomial error structure and log-link function were used to compare trap abundance according to LED colour, meteorological conditions and seasonality.

Results: The experiment was conducted over 49 nights with 42,766 Culicoides caught in 329 collections. Culicoides obsoletus Meigen and Culicoides scoticus Downes and Kettle responded indiscriminately to all wavelengths of LED used with the exception of red which was significantly less attractive. In contrast, Culicoides dewulfi Goetghebuer and Culicoides pulicaris Linnaeus were found in significantly greater numbers in the green LED trap than in the UV LED trap. The LED traps collected significantly fewer Culicoides than the standard CDC UV light-suction trap.

Conclusions: Catches of Culicoides were reduced in LED traps when compared to the standard CDC UV trap, however, their reduced power requirement and small size fulfils a requirement for trapping in logistically challenging areas or where many traps are deployed at a single site. Future work should combine light wavelengths to improve trapping sensitivity and potentially enable direct comparisons with collections from hosts, although this may ultimately require different forms of baits to be developed.
\end{abstract}

Keywords: Ceratopogonidae, Bluetongue virus, Schmallenberg virus, Orbivirus, Surveillance, Diptera, Culicoides

\footnotetext{
* Correspondence: andrew.hope@lstmed.ac.uk

${ }^{1}$ Vector-borne Viral Disease Programme, The Pirbright Institute, Pirbright,

Surrey, United Kingdom

Full list of author information is available at the end of the article
} 


\section{Background}

Culicoides biting midges (Diptera: Ceratopogonidae) are responsible for the transmission of economically important arboviruses including bluetongue virus (BTV), African horse sickness virus (AHSV) and the newly emerged Schmallenberg virus (SBV) [1,2]. An accurate assessment of the phenology, abundance and distribution of Culicoides is important for understanding the potential transmission of BTV, AHSV and SBV and in monitoring disease outbreaks [3]. As a result, large-scale monitoring of Culicoides populations is carried out in many European countries and these are entirely reliant on overnight collections from ultraviolet light-suction (UVLS) traps of various designs [4]. In addition, UVLS traps have also been used in a vast array of behavioural studies across the region at a variety of spatial scales (e.g. between farm: [5]; within farms: [6-8]), largely in response to the incursions of BTV and SBV into Europe. While these traps are known to be unrepresentative of host biting rates on livestock in the region [9-11], they provide a rapid and logistically straightforward means of sampling Culicoides populations.

In the early stages of systematic Culicoides surveillance in southern Europe there was a divergence in the design of UVLS traps used in monitoring. In Italy [12], France [13], Switzerland $[14,15]$ and many other countries, the Onderstepoort Veterinary Institute (OVI) trap design was used. This is a robust trap design weighing over $4 \mathrm{~kg}$ without a battery that is baited with an 8 watt $30 \mathrm{~cm}$ UV tube and uses an $11 \mathrm{~cm}$ diameter fan for suction. The OVI UVLS traps has been demonstrated to catch vast numbers of some species (e.g. over one million Culicoides imicola Kieffer have been trapped in a single night in South Africa [16]) and, anecdotally, has a strong reliability record in the case of the mains electricity powered model. There are issues, however, both in the commercial production of the OVI light-suction trap for what is a very limited market (these traps are used solely for Culicoides surveillance) and in the use of multiple traps for behavioural studies in the field due to its bulk and mains power requirements.

The Centre for Disease Control (CDC) UVLS trap (design 1212 and the similar 912) is also used for monitoring Culicoides populations in large-scale surveillance schemes, particularly in Spain [17]. This trap is baited with a $4 \mathrm{~W}$ $13.5 \mathrm{~cm}$ UV tube and uses an $8 \mathrm{~cm}$ fan for suction resulting in an overall power consumption of approximately $0.86 \mathrm{amps} /$ hour (equating to a single night of trapping using a 6 volt/10 amp hours battery). The CDC UVLS (1212) is substantially lighter in weight than the OVI UVLS, weighing approximately $750 \mathrm{~g}$ excluding battery and hence more suitable for deployment in behavioural studies on a local scale. This trap is often used for large-scale monitoring of mosquitoes and hence is commercially sustainable. The CDC UVLS (1212) trap has, not surprisingly, been demonstrated to catch fewer Culicoides than the OVI UVLS trap, presumably due to its less powerful light bait and suction $[18,19]$.

The development of low-cost light emitting diodes (LEDs) as a source of light has provided an opportunity to address some of the deficiencies of conventional light baits [20]. A major advantage in using LEDs is the reduced power consumption of these light sources when compared with standard incandescent and UV light sources. The wide range of applications for these traps has also enabled commercial development, although use remains limited at present in comparison to conventional UVLS traps. At present the Bioquip model 2770 represents the extreme of increased portability and reduced power consumption, based on an original design trialled on phlebotomine sand flies [21]. This trap weighs $<500 \mathrm{~g}$ without a battery and operates with $8 \mathrm{LED}$ elements to provide $360^{\circ}$ coverage, but only in a horizontal plane, with a viewing angle of $45^{\circ}$. Power draw, even in comparison to the CDC UVLS (1212) trap, is minimal with a consumption of 0.35 amps/ hour, (allowing three nights of trapping to be conducted with a single 6 Volt/10 amp hours battery charge). The unit can also be customised with LEDs of specific wavelength allowing certain species with preferences for a specific wavelength to be targeted.

Field studies have already used LEDs to assess the response of Culicoides to different wavelengths of light. LED-baited LS traps are currently used for surveillance of Culicoides in Australia, prompted by the appearance of Akabane virus in areas lacking the principal vector $C$. brevitarsis Kieffer in incandescent light-baited trap catches [22]. This observation led to a hypothesis that $C$. brevitarsis was under-represented by this trapping method and a green LED baited trap was subsequently found to collect significantly greater numbers, in addition to a greater diversity of Culicoides species than the standard incandescent control or blue- or UV-LED baited traps [23]. As a result of these findings, and the fact that LED-based traps consume less power making trapping logistics more straightforward, the Australian National Arbovirus Monitoring Program now employs green LED traps for monitoring C. brevitarsis in low density areas [24].

In this trial we examine the potential to use commercially available and highly portable LED-baited light-suction traps to collect Culicoides on a farm in the UK. Different light wavelength modules are used to investigate their impact on species diversity and abundance, and compared with a standard CDC UVLS. Conclusions regarding the possible role for LED-baited traps are then made along with potential for further improvement as tools to study Culicoides behaviour.

\section{Methods \\ Study site}

The trial was conducted from May to September 2011 at a farm in Surrey, south east England (51 20 $09.60^{\prime \prime}$, 
$\left.0^{\circ} 33^{\prime} 55.87^{\prime \prime} \mathrm{W}\right)$. The site comprised a large field $(140 \mathrm{~m} \times 120 \mathrm{~m})$ subdivided into smaller grazing enclosures that in total accommodated four horses and two pigs. Two sides of the site were surrounded by deciduous woodland and two sides bordered further grazing land used for horses.

\section{Collection methods}

Culicoides were collected using LS traps (Model 2770, Bioquip Inc., USA) fitted with LED platforms consisting of 8 individual LEDs emitting at specific wavelengths [20]. Six different colours of LED were used in the traps (Figure 1): ultraviolet (390 nm); Blue (430 nm); Green (570 nm); Yellow (590 nm); Red (660 nm) and White (425 nm - $750 \mathrm{~nm}$ with peaks at $450 \mathrm{~nm}$ and $580 \mathrm{~nm}$ ). An additional standard CDC LS trap (Model 912, J. W. Hock, USA) fitted with a 4 watt UV tube (320-420 nm) was used as a positive control. Traps were hung at a height of $1.5 \mathrm{~m}$, with an intertrap distance of at least $50 \mathrm{~m}$. Culicoides attracted to the light traps were collected into beakers containing $200 \mathrm{ml}$ of water and a drop of detergent. Following collection samples were drained through a sieve and transferred to $70 \%$ ethanol for storage.
Traps using 6 volt batteries were operated from late afternoon until the following morning in order to encompass the sunset and sunrise peaks in UK Culicoides activity [25]. On night 1 the trap treatments were randomly assigned to locations and on subsequent nights the treatments were rotated to the next location in a clockwise direction. After seven nights of trapping, all traps had collected at each of the seven locations and the treatments were again re-randomised to trap locations for the start of the next rotation. A total of seven rotations were completed giving 49 nights of data collection.

\section{Meteorological data}

Meteorological data were collected throughout the sampling period using an automatic weather station (CR800 data logger, Campbell Scientific, UK). Data collected were: air temperature $\left({ }^{\circ} \mathrm{C}\right)$; relative humidity (\%); solar intensity $\left(\mathrm{Wm}^{-2}\right)$; wind speed $\left(\mathrm{ms}^{-1}\right)$ and wind direction $\left(^{\circ}\right)$. For data analysis, wind direction was transformed using the ArcTangent 2 function in Excel as it is a circular variable and therefore wind direction at $0^{\circ}$ and $360^{\circ}$ represent the same direction.
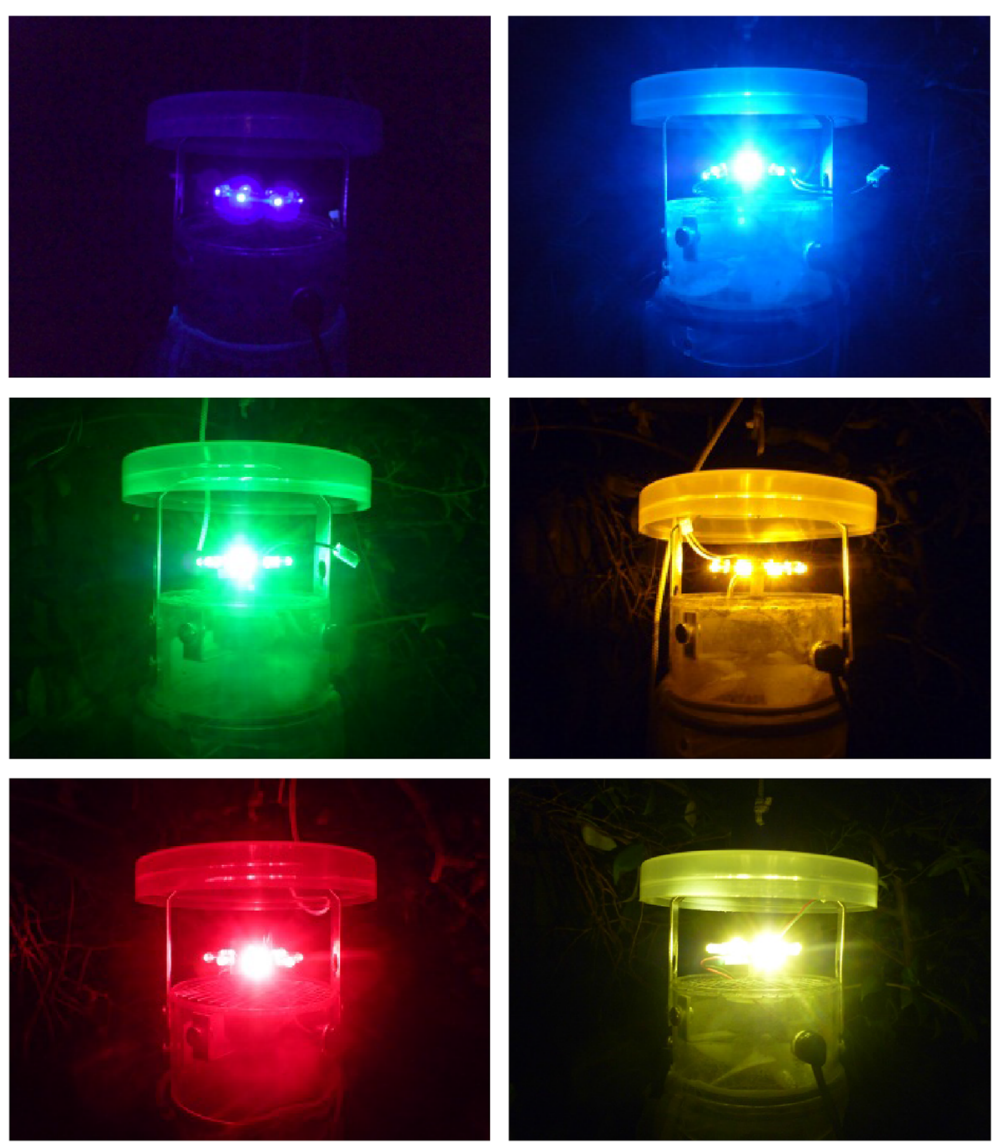

Figure 1 Light emitting diode baits used during investigation of differential attraction of Culicoides to commercially produced light-suction traps. Colours used were: UV (390 nm), Blue (430 nm), Green (570 nm), Yellow (590 nm), Red (660 nm) and White (427-750 nm). 


\section{Sample identification}

Trapped Culicoides were initially sorted using morphological characteristics according to physiological status into unpigmented, pigmented, gravid and blood-fed individuals. While $C$. chiopterus Meigen was identified by morphology (small size and pale markings on wings), other female members of the subgenus Avaritia (C. obsoletus, C. scoticus and C. dewulfi) were identified using a modified multiplex polymerase chain reaction (PCR) $[26,27]$. To extract DNA, Culicoides were removed from $70 \%$ ethanol storage and allowed to dry on paper towelling for 10 minutes, before being placed individually into $2 \mathrm{ml}$ micro-collection tubes (Qiagen, UK). Ten microlitres of $2 \%$ proteinase-k (Bioline, UK) (made in solution with tris calcium acetate) was then added, along with $200 \mu \mathrm{l}$ of $5 \%$ chelex (Bio-Rad, UK). Samples were homogenised in two cycles of two minutes at $25 \mathrm{~Hz}$ in a Tissuelyser (Qiagen, UK) and then incubated overnight at $37^{\circ} \mathrm{C}$. Following incubation, $4 \mu \mathrm{l}$ of each sample was removed and added to a PCR plate (Abgene, UK). Samples were subjected to an eight minute cycle at $99^{\circ} \mathrm{C}$ to de-activate the proteinase-k. PCR mastermix for each PCR plate consisted: $25 \mu \mathrm{l}$ of $10 \mu \mathrm{M}$ forward primer specific to each species $(C$. obsoletus: TGCAGGAGCTTCTGTAGATTTG; C. scoticus: ACCGGCATAACTTTTGATCG; C. dewulfi: ATACTAGG AGCGCCCGACAT) [26]; $25 \mu \mathrm{l}$ DNAse free water; $100 \mu \mathrm{l}$ of $10 \mu \mathrm{M}$ universal reverse primer (CAGGTAAAATT AAAATATAAACTTCTGG) [27]; $7 \mu \mathrm{l} \quad \mathrm{MgCl}_{2}$ solution (Bioline, UK) and $400 \mu \mathrm{l}$ Biomix Red solution (Bioline, UK).

Each $4 \mu \mathrm{l}$ sample of extracted DNA had $6 \mu \mathrm{l}$ of mastermix added. In addition to the test samples, each plate also contained 3 positive controls, using DNA extracted from males of each species using spin column methods according to the manufacturer's protocol (Qiagen, UK), and 3 negative controls, which consisted of $4 \mu \mathrm{l}$ of DNAse free water. Samples were then placed in a thermocycler with the following profile: initial denaturing step at $94^{\circ} \mathrm{C}$ for 4 minutes; 32 cycles of $94^{\circ} \mathrm{C}$ for 30 seconds, $60^{\circ} \mathrm{C}$ for 30 seconds, $72^{\circ} \mathrm{C}$ for 1 minute; followed by a final extension step at $72^{\circ} \mathrm{C}$ for 5 minutes [27]. Polymerase chain reaction products were examined by electrophoresis using $2 \%$ agarose e-gels (Invitrogen, UK) and these were compared to a DNA ladder (Invitrogen, UK) for means of identification.

Due to the large number of Culicoides collected it was not possible to process all individuals to species level using the diagnostic PCR and a sub-sampling method was used. For each trap treatment, two nights were randomly selected from each seven nights rotation and all C. obsoletus/C. scoticus/C.dewulfi females within the trap catch were identified by PCR to species level. The PCR results were then combined and for each seven night rotation the proportions of each species were applied to the collections from the remaining five nights of the rotation. If collections failed to amplify then another night was randomly selected for analysis and failed samples were excluded from final estimates.

\section{Statistical analysis}

Generalised linear models (GLMs) with negative binomial error structure and log-link function were constructed in $\mathrm{R}$ (version 2.15.2) to compare Culicoides collections according to the wavelengths of light used as bait. Initial GLMs included all meteorological variables: air temperature $\left({ }^{\circ} \mathrm{C}\right)$; relative humidity (\%); solar radiation $\left(\mathrm{Wm}^{-2}\right)$; wind speed $\left(\mathrm{ms}^{-1}\right)$; transformed wind direction and variation in wind direction $\left({ }^{\circ}\right)$. Linear and quadratic temporal trends were included to model the effect of seasonality on collections. Trap site was included as an explanatory variable to account for any variation between the seven trap locations. The construction of final models was preceded by stepwise deletion of non-significant $(p>0.05)$ variables, with the final model corresponding to the one where all terms were significant. Differences in mean trap catch size between the different traps were then assessed using Tukey's allpairs comparison test.

\section{Results}

Sampling was conducted over 49 nights, giving a total of 329 successful collections, after the exclusion of 14 trap failures $(4.1 \%$ of total catches: 12 due to motor failures on LED traps; 1 due to battery failure on CDC LS trap; 1 due to operator error). A total of 42,766 Culicoides were collected, the majority of which were females of $C$. obsoletus, C. scoticus and C. dewulfi, accounting for $84.3 \%$ of the trap collection (Table 1). Males of these species were far less commonly caught, constituting $3.2 \%$ of the total collection. Other species collected in order of abundance were C. brunnicans Edwards (4.8\%); C. pulicaris (2.1\%); C. punctatus Meigen (0.9\%); and C. impunctatus Goetghebuer (0.8\%). The remaining 3.9\% of individuals included rarer species (C. achrayi Kettle and Lawson, C. festivipennis Kieffer, C. pictipennis Staeger, C. nubeculosus Meigen and C. chiopterus). The species diversity of Culicoides collected per trap according to light wavelength are presented in Table 1.

A total of 9,918 female individuals identified morphologically as being one of either C. obsoletus, C. scoticus or $C$. dewulfi were subjected to molecular identification by multiplex PCR, of which $88.9 \%$ were successfully identified and $11.1 \%$ failed due to poor DNA extraction. Of the 8,853 individuals successfully identified, 5,862 (66.2\%) were C. obsoletus, 2,789 (31.5\%) were C. scoticus and $202(2.3 \%)$ were $C$. dewulfi. The sub-sampling results were then used to calculate the total numbers for these species per physiological status as shown in Table 2; samples that failed to amplify in the PCR are 
Table 1 Culicoides biting midges collected during a comparative study of commercially available light emitting diode baited suction traps. Studies were conducted over a total of $\mathbf{4 9}$ days at a farm holding in the United Kingdom

\begin{tabular}{|c|c|c|c|c|c|c|c|c|}
\hline \multirow[t]{2}{*}{ Species } & \multicolumn{7}{|c|}{ Total Culicoides collected (Mean \pm SEM) } & \multirow[b]{2}{*}{ Total $(n=329)$} \\
\hline & $\operatorname{CDC}(n=48)$ & Ultraviolet $(n=47)$ & Blue $(n=46)$ & Green $(n=49)$ & Yellow $(n=48)$ & $\operatorname{Red}(n=45)$ & White $(n=46)$ & \\
\hline C. obsoletus/C. scoticus/C. dewulfi & $20,569(429 \pm 110)$ & $3,077(65.5 \pm 18.6)$ & $3,515(76.4 \pm 24.1)$ & $3,965(80.9 \pm 17.3)$ & $2,810(58.5 \pm 25.0)$ & $122(2.7 \pm 0.6)$ & $3,379(73.5 \pm 23.0)$ & 37,437 \\
\hline C. pulicaris & $389(8.1 \pm 2.8)$ & $49(1.0 \pm 0.3)$ & $119(2.6 \pm 0.9)$ & $157(3.2 \pm 1.0)$ & $69(1.4 \pm 0.5)$ & $1(0.02 \pm 0.0)$ & $100(2.2 \pm 0.7)$ & 884 \\
\hline C. punctatus & $210(4.4 \pm 1.7)$ & $20(0.4 \pm 0.2)$ & $55(1.2 \pm 0.9)$ & $77(1.6 \pm 0.5)$ & $31(0.6 \pm 0.3)$ & 0 & $13(0.3 \pm 0.1)$ & 406 \\
\hline C. impunctatus & $93(1.9 \pm 0.8)$ & $54(1.1 \pm 0.6)$ & $91(2.0 \pm 1.2)$ & $72(1.5 \pm 0.4)$ & $16(0.3 \pm 0.1)$ & $2(0.04 \pm 0.0)$ & $16(0.3 \pm 0.1)$ & 344 \\
\hline C. brunnicans & $264(12.6 \pm 4.9)$ & $103(4.9 \pm 1.9)$ & $542(25.8 \pm 22.4)$ & $744(35.3 \pm 27.3)$ & $213(10.1 \pm 5.3)$ & $19(1.0 \pm 0.4)$ & $186(10.3 \pm 5.5)$ & 2,071 \\
\hline Other Species & 527 & 154 & 319 & 357 & 164 & 3 & 100 & 1,624 \\
\hline Total & 22,052 & 3,457 & 4,641 & 5,372 & 3,303 & 147 & 3,794 & 42,766 \\
\hline
\end{tabular}


Table 2 Estimated abundances of $C$. obsoletus, C. scoticus and C. dewulfi based on multiplex polymerase chain reaction identification of subsamples

\begin{tabular}{|c|c|c|c|c|c|c|c|c|c|}
\hline Species & Physiological status & $\mathrm{CDC}$ & UV & Blue & Green & Yellow & Red & White & Total \\
\hline \multirow[t]{6}{*}{ C. obsoletus } & Unpigmented & 7,336 & 1,259 & 1,351 & 1,628 & 979 & 24 & 1,458 & 14,035 \\
\hline & Pigmented & 2,545 & 604 & 752 & 814 & 529 & 21 & 537 & 5,802 \\
\hline & Blood-fed & 92 & 9 & 44 & 72 & 46 & 0 & 96 & 359 \\
\hline & Gravid & 219 & 42 & 153 & 189 & 123 & 1 & 27 & 754 \\
\hline & Male & 384 & 62 & 97 & 209 & 37 & 8 & 88 & 885 \\
\hline & Total & 10,576 & 1,976 & 2,397 & 2,912 & 1,714 & 54 & 2,206 & 21,835 \\
\hline \multirow[t]{6}{*}{ C. scoticus } & Unpigmented & 4,826 & 489 & 301 & 316 & 552 & 28 & 381 & 6,893 \\
\hline & Pigmented & 4,405 & 425 & 307 & 441 & 310 & 21 & 252 & 6,161 \\
\hline & Blood-fed & 3 & 1 & 7 & 17 & 4 & 0 & 12 & 44 \\
\hline & Gravid & 96 & 6 & 28 & 3 & 9 & 1 & 30 & 173 \\
\hline & Male & 264 & 24 & 41 & 56 & 37 & 3 & 24 & 449 \\
\hline & Total & 9,594 & 945 & 684 & 833 & 912 & 53 & 699 & 13,720 \\
\hline \multirow[t]{6}{*}{ C. dewulfi } & Unpigmented & 147 & 14 & 45 & 19 & 33 & 0 & 40 & 298 \\
\hline & Pigmented & 92 & 7 & 70 & 36 & 22 & 2 & 37 & 266 \\
\hline & Blood-fed & 2 & 0 & 37 & 3 & 0 & 0 & 0 & 42 \\
\hline & Gravid & 86 & 19 & 13 & 38 & 1 & 0 & 1 & 158 \\
\hline & Male & 17 & 6 & 2 & 10 & 3 & 2 & 6 & 46 \\
\hline & Total & 344 & 46 & 167 & 106 & 59 & 4 & 84 & 810 \\
\hline Total & & 20,514 & 2,967 & 3,248 & 3,851 & 2,685 & 111 & 2,989 & 36,365 \\
\hline
\end{tabular}

not included and six males belonging to the subgenus Avaritia are also excluded as these were damaged and could not be identified to species level. Numbers of $C$. obsoletus and C. scoticus females collected were sufficient for three models for each species to be constructed for analysis: total females (includes all physiological states); unpigmented females and pigmented females. For C. dewulfi, C. pulicaris and C. brunnicans, analyses were restricted to single models for total females of all physiological states due to the smaller number of individuals collected. Full details of the final models for each species are presented in Additional file 1: Tables S1-S6.

After allowing for the effects of meteorological variables, trap location and temporal trends the CDC trap collected significantly more C. obsoletus and C. scoticus of all physiological types than any of the other traps $(\mathrm{p}<0.01)$ (S.2. and S.4.). The one exception was for the collection of pigmented $C$. obsoletus using the greenwavelength light-suction trap where there was no statistical difference with the CDC trap, although this result was marginal $(p=0.057)$. Furthermore, the red trap collected significantly fewer individuals than all other traps $(\mathrm{p}<0.001)$.

CDC UVLS trap collections of $C$. dewulfi were not significantly different to the blue and green LED LS traps $(\mathrm{p}>0.05)$ (Additional file 1: Table S6). Amongst the LED light-suction traps there were no significant differences between the blue and green-baited LS traps, but both collected significantly greater numbers than the UV, yellow and red LED LS traps $(\mathrm{p}<0.05)$. The red LED baited LS trap collected significantly fewer individuals than all other LS traps $(\mathrm{p}<0.05)$.

Analysis of $C$. pulicaris collections showed a similar pattern to $C$. dewulfi with the CDC UVLS trap collections not being significantly greater in abundance than the blue and green LED baited LS trap collections ( $p>0.05)$ and no difference was observed between the blue and green-baited LED LS traps (Additional file 1: Table S6). The green-baited LED LS trap also collected significantly greater numbers than the UV, yellow and red LED baited LS traps $(\mathrm{p}<0.01)$ and the red-baited LED LS trap consistently collected fewer $C$. pulicaris than all other LS traps. In the $C$. brunnicans analysis there was also no difference in collections made using the CDC UVLS trap compared to the green and blue LED baited LS traps and there was also no difference to the white LED baited LS trap $(p<0.05)$ (see Additional file 1: Tables S5 and S6.). Among the LED-baited LS traps no differences were observed between the blue, green and UV-baited LED LS traps, but the red LED LS trap collected significantly fewer $C$. brunnicans than all other traps with the exception of the UV LED LS trap 
where no significant difference was found. This species was restricted to the early part of the season in contrast to the other abundant species that were present throughout the trial (Figure 1).

Collections of all species made at trap location 1 were typically higher than at other locations though the differences were not always statistically significant (see Additional file 1: Tables S1, S3 and S5). Where statistically significant the effects of meteorological variables were consistent across species (see Additional file 1: Tables S1, S3 and S5). Higher catches were associated with higher temperatures, higher humidity, lower solar radiation and lower wind-speeds. Seasonal variation in populations was marked, with C. brunnicans activity restricted to the beginning of the adult season, while other species were present throughout the sampling period (Figure 2).
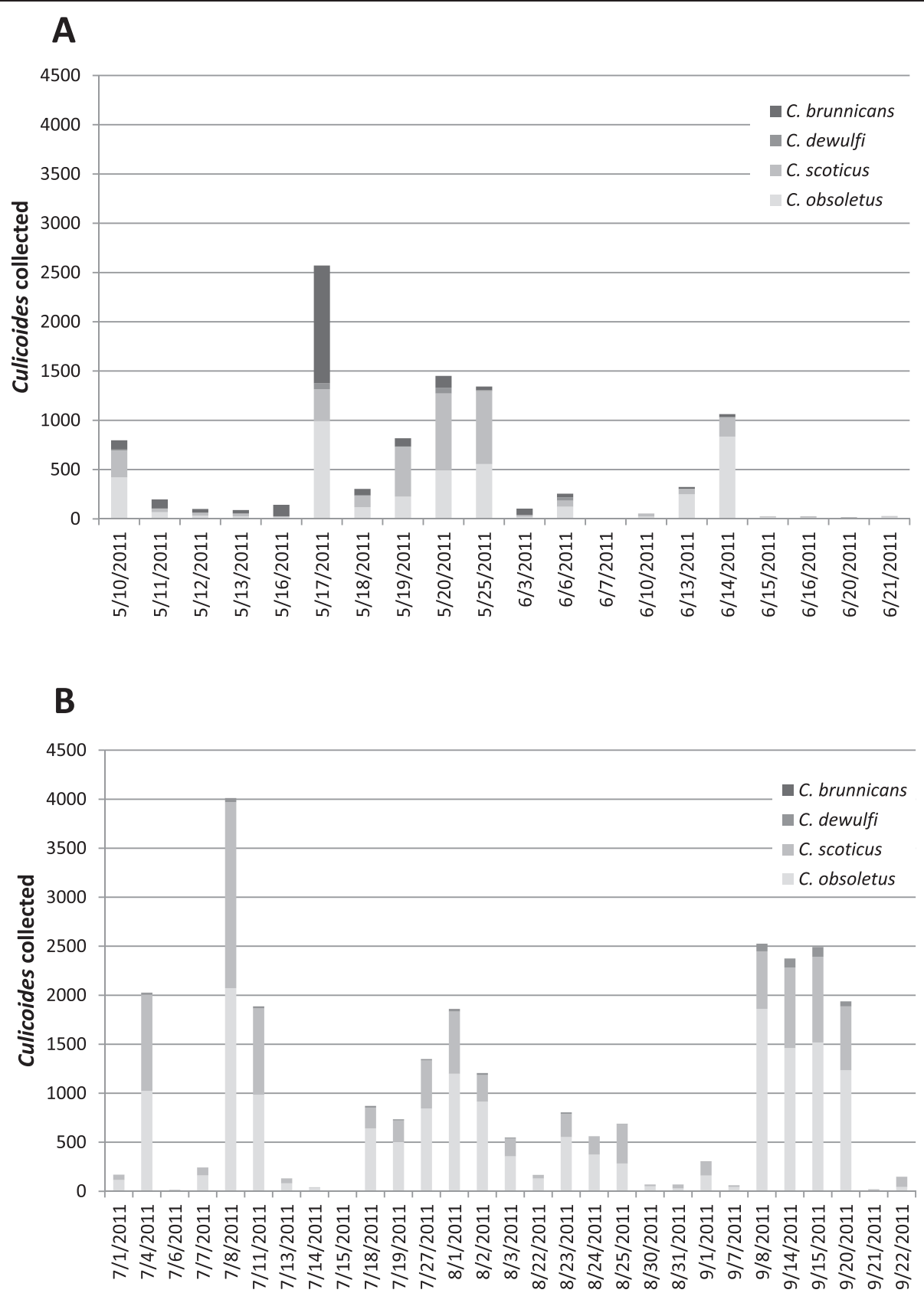

Figure 2 Seasonal occurrence of common Culicoides species from (A) May to June and (B) July to September. Collections are pooled across suction trap catches using light emitting diodes and a conventional ultraviolet light baited trap. Abundance for C. obsoletus, C. scoticus and C. dewulfi is calculated from a subsample of specimens identified to species level using a multiplex polymerase chain reaction assay while $C$. brunnicans were identified directly using their morphology. 


\section{Discussion}

This is the first study to assess the performance of LEDbaited LS traps in direct comparison to a standard surveillance tool for European Culicoides species. Throughout the study, the CDC UVLS trap (912) consistently outperformed the LED LS traps in the abundance of all Culicoides species collected, with the exception of $C$. brunnicans. Despite this, numbers collected by the LED LS traps were still substantial, with maximum single night catches exceeding 500 individuals for blue, green and white LED baits. Trap reliability, while poorer than that of the bulkier traps, was manageable across the field season and the reduced requirement for battery charging made carrying out the trapping substantially more straightforward. LED LS traps may therefore play a role in studies requiring intensive trapping at a single site where the daily recharging of batteries is prohibitively time consuming. A second potential role the LED LS traps could fulfil is in sampling of Culicoides populations prior to the establishment of major surveillance efforts, particularly in regions with limited, or intermittent, supply of mains electricity. Here, their portability would be of great use in reducing luggage weight and reduce power requirements which may enable sampling missions to be extended in the field.

Across the wavelengths of light tested, the most consistent pattern of Culicoides response was a poor attraction to the red $(660 \mathrm{~nm})$ LED LS trap. Excluding the red LED LS trap, C. obsoletus and C. scoticus appeared to exhibit an indiscriminate response to the different LED LS traps used. In contrast, while $C$. dewulfi was collected in fewer numbers than $C$. obsoletus and $C$. scoticus, the blue and green LED LS traps collected significantly more than the UV LED LS trap. This demonstrates that $C$. dewulfi shows differential attraction to traps emitting different wavelengths and that the use of UV light baited traps may risk under-estimating the population of this species. Very similar results were also found in the collection of C. pulicaris and C. brunnicans. All of these species demonstrated parallels to C. brevitarsis in Australia which was previously found to respond to green LED baits [23]. While the experimental design used a clockwise rotation of traps rather than a more ideal randomised allocation of placement it is assumed that this did not greatly influence results due to the high degree of replication (7 full rotations with random initial allocation of sites).

While the relative influence of each northern European species of Culicoides in transmission of BTV and SBV remains uncertain $[2,28]$, it is unlikely that these differences are sufficient to warrant alteration of light wavelengths used for standard surveillance. As only commercially available traps were used in the present study no attempt was made to standardise for the intensity of light emitted by each trap. Further investigation using LEDs with a uniform light intensity would reveal whether or not the green wavelength is inherently superior to UV for trapping $C$. dewulfi, C. pulicaris and C. brunnicans. A separate laboratory study to investigate spectral sensitivity through electroretinograms as conducted with sandflies may also yield useful information on the response of Culicoides to different light wavelengths [29]. An additional option for increasing the sensitivity of catches would be to combine LEDs of different wavelengths within a single unit. This could have the advantage of increased detection of green/blue sensitive species, which at present may be under-represented in UV LS trap catches.

The study site chosen for the trial contained large populations of most of the common livestock-associated species of Culicoides in the UK, confirmed through the use of the control CDC light-suction trap. These collections were dominated by $C$. obsoletus and $C$. scoticus, which are ubiquitous across Europe, with a lesser abundance of $C$. dewulfi, C. brunnicans, C. pulicaris, C. punctatus and C. impunctatus, all of which have been recorded in previous trials carried out locally to this region [30]. It was notable, however, that cattle-dung breeding species (namely $C$. dewulfi and C. chiopterus) were under-represented as a proportion of total catch when compared to farm studies conducted elsewhere in northern Europe [31]. This may have been due to the close relationship between these species and cattle [32], which were not directly present at the site during the trial (although they were occasionally grazed in adjacent fields to the study area). As expected, flight behaviour of Culicoides was heavily influenced by meteorological variables making it essential to include these data in any analysis of field collections of these species $[9,33]$. The models generated are broadly in agreement with previous Culicoides studies in showing that temperature and humidity have a positive impact on trap collections whereas wind speed has a negative impact.

A challenge for interpretation of LS trap catches of Culicoides is in assessing the abundance of individuals collected against those biting hosts. There is clear evidence that this relationship is both complex and varies considerably from study to study [9-11]. Comparative studies with other and combined LED wavelengths may yield improvements in this comparability; however, the intrinsic limitation of light based sampling methods may require alternative methods to be used, not least the fact that light traps are effective for only part of the adult active period of many Culicoides species. These methods could include the use of semiochemical-based approaches as already trialled in northern Europe that mimic host location cues, although these remain at a relatively early stage of development [34].

\section{Conclusions}

The abundance of Culicoides collected was reduced in the LED LS traps when compared to the standard CDC 
UV LS trap, however, their reduced power requirement and small size fulfils a requirement for trapping in logistically challenging areas or where many traps are deployed at a single site. Future work should consider combining light wavelengths which may improve trapping sensitivity and direct comparisons with collections from hosts to examine comparability with biting rates.

\section{Additional file}

Additional file 1: Table S1. Regression coefficients for final models to describe collections of C. obsoletus. Table S2. Multiple Tukey's all-pair comparisons of catch size between trap types for C. obsoletus females. Table S3. Regression coefficients for final models to describe collections of C. scoticus. Table S4. Multiple Tukey's all-pair comparisons of catch size between trap types for $\mathrm{C}$. scoticus females. Table S5. Regression coefficients for final models of females of C. dewulfi, C. pulicaris and C. brunnicans. Table S6. Multiple Tukey's all-pair comparisons of catch size between trap types for C. dewulfi, C. pulicaris and C. brunnicans females.

\section{Competing interests}

The authors declare that they have no competing interests.

\section{Authors' contributions}

AH, SC, SG, MB and CS designed the study. AH, FS, ED and JB performed the experiments. All authors read and approved the final version of the manuscript.

\section{Acknowledgements}

The authors would like to acknowledge Lara Harrup, Karin Darpel and Eva Veronesi for discussions regarding field sampling and the molecular processing in the study and Paul Phipps for providing access to the site where the study was conducted. AH was funded by a BBSRC Doctoral Training Studentship: BBF0164921. FS \& SC were funded by the UK National Culicoides Laboratory.

\section{Author details}

${ }^{1}$ Vector-borne Viral Disease Programme, The Pirbright Institute, Pirbright, Surrey, United Kingdom. ${ }^{2}$ Liverpool University Climate and Infectious Diseases of Animals (Lucinda) Group, University of Liverpool, Neston, Cheshire, United Kingdom. ${ }^{3}$ Health Protection Research Unit in Emerging and Zoonotic Infections, University of Liverpool, Liverpool, United Kingdom.

Received: 27 October 2014 Accepted: 8 April 2015

Published online: 22 April 2015

\section{References}

1. Hoffmann B, Scheuch M, Hoeper D, Jungblut R, Holsteg M, Schirrmeier H, et al. Novel Orthobunyavirus in Cattle, Europe, 2011. Emerg Infect Dis. 2012;18(3):469-72

2. Carpenter S, Wilson A, Mellor PS. Culicoides and the emergence of bluetongue virus in northern Europe. Trends Microbiol. 2009;17(4):172-8.

3. Gubbins S, Carpenter S, Baylis M, Wood JLN, Mellor PS. Assessing the risk of bluetongue to UK livestock: uncertainty and sensitivity analyses of a temperature-dependent model for the basic reproduction number. J R Soc Interface. 2008:5(20):363-71.

4. Mellor PS, Tabachnick W, Baldet T, Baylis M, Bellis G, Calistri P, et al. Conclusions of working groups. Group 2. Vectors. Proceedings of the 3rd OIE Bluetongue International Symposium, 26-29 October 2003, Taormina, Italy. Vet Italia. 2004:40:715-7.

5. Kluiters G, Sugden D, Guis H, McIntyre KM, Labuschagne K, Vilar MJ, et al Modelling the spatial distribution of Culicoides biting midges at the local scale. J Appl Ecol. 2013;50(1):232-42.

6. Kirkeby C, Bodker R, Stockmarr A, Lind P. Spatio-temporal optimization of sampling for bluetongue vectors (Culicoides) near grazing animals. Parasit Vectors. 2013;6:151.
7. Kirkeby C, Bodker R, Stockmarr A, Lind P. Spatial abundance and clustering of Culicoides (Diptera: Ceratopogonidae) on a local scale. Parasit Vectors. 2013;6:43.

8. Baylis M, Parkin H, Kreppel K, Carpenter S, Mellor PS, Mclntyre KM. Evaluation of housing as a means to protect cattle from Culicoides biting midges, the vectors of bluetongue virus. Med Vet Entomol. 2010;24(1):38-45.

9. Carpenter S, Szmaragd C, Barber J, Labuschagne K, Gubbins S, Mellor P. An assessment of Culicoides surveillance techniques in northern Europe: have we underestimated a potential bluetongue virus vector? J Appl Ecol. 2008:45(4):1237-45.

10. Viennet E, Garros C, Gardes L, Rakotoarivony I, Allene X, Lancelot R, et al. Host preferences of Palaearctic Culicoides biting midges: implications for transmission of orbiviruses. Med Vet Entomol. 2013;27(3):255-66.

11. Gerry AC, Monteys VSI, Vidal JOM, Francino O, Mullens BA. Biting rates of Culicoides midges (Diptera: Ceratopogonidae) on sheep in Northeastern Spain in relation to midge capture using UV light and carbon dioxidebaited traps. J Med Entomol. 2009;46(3):615-24.

12. Goffredo M, Meiswinkel R. Entomological surveillance of bluetongue in Italy: methods of capture, catch analysis and identification of Culicoides biting midges. In: MacLachlan NJ, Pearson JE, editors. Bluetongue, Pt 1, Proceedings, vol. 40. 2004. p. 260-5.

13. Venail R, Balenghien T, Guis H, Tran A, Setier-Rio M-L, Delecolle JC, et al. Assessing diversity and abundance of vector populations at a national scale: example of Culicoides surveillance in France after bluetongue virus emergence. In: Melhlhorn $\mathrm{H}$, editor. Arthropods as vectors of emerging diseases, parasitology research monographs 3. Berlin, Heidelberg: Springer-Verlag; 2012. p. 77-102.

14. Kaufmann C, Steinmann IC, Hegglin D, Schaffner F, Mathis A. Spatiotemporal occurrence of Culicoides biting midges in the climatic regions of Switzerland, along with large scale species identification by MALDI-TOF mass spectrometry. Parasit Vectors. 2012;5:10.

15. Cagienard A, Griot C, Mellor PS, Denison E, Stark KDC. Bluetongue vector species of Culicoides in Switzerland. Med Vet Entomol. 2006;20(2):239-47.

16. Meiswinkel R, Venter GJ, Nevill EM. Vectors: Culicoides spp. Oxford, UK: Oxford University Press; 2004.

17. Calvete C, Miranda MA, Estrada R, Borras D, Monteys VSI, Collantes F, et al. Spatial distribution of Culicoides imicola, the main vector of bluetongue virus, in Spain. Vet Rec. 2006;158(4):130-1.

18. Venter GJ, Labuschagne K, Boikanyo SNB, Morey L. The suitability of the Triple trap for the collection of South African livestock-associated Culicoides species. J S African Vet Assoc. 2013; 84(1):Art. \#994-Art. \#994.

19. Del Rio R, Monerris M, Miquel M, Borras D, Calvete C, Estrada R, et al. Collection of Culicoides spp. with four light trap models during different seasons in the Balearic Islands. Vet Parasitol. 2013;195(1-2):150-6.

20. Cohnstaedt LW, Gillen Jl, Munstermann LE. Light-emitting diode technology improves insect trapping. J Am Mosq Cont Assoc. 2008;24(2):331-4.

21. Cohnstaedt LW, Rochon K, Duehl AJ, Anderson JF, Barrera R, Su N-Y, et al. Arthropod surveillance programs: basic components, strategies, and analysis. Ann Entomol Soc Am. 2012;105(2):135-49.

22. Bishop AL, Worrall RJ, Spohr LJ, McKenzie HJ, Barchia IM. Improving light-trap efficiency for Culicoides spp. with light-emitting diodes. In: MacLachlan NJ, Pearson JE, editors. Bluetongue, Pt 1, Proceedings, vol. 40. 2004. p. 266-9.

23. Bishop AL, Worrall R, Spohr LJ, McKenzie HJ, Barchia IM. Response of Culicoides spp. (Diptera: Ceratopogonidae) to light-emitting diodes. Aus J Entomol. 2004;43:184-8.

24. Bishop AL, Bellis GA, McKenzie HJ, Spohr LJ, Worrall RJ, Harris AM, et al. Light trapping of biting midges Culicoides spp. (Diptera: Ceratopogonidae) with green light-emitting diodes. Aus J Entomol. 2006;45:202-5.

25. Sanders CJ, Gubbins S, Mellor PS, Barber J, Golding N, Harrup LE, et al. Investigation of diel activity of Culicoides biting midges (Diptera: Ceratopogonidae) in the United Kingdom by using a vehicle-mounted trap. J Med Entomol. 2012;49(3):757-65.

26. Nolan DV, Carpenter S, Barber J, Mellor PS, Dallas JF, Mordue AJ, et al. Rapid diagnostic PCR assays for members of the Culicoides obsoletus and Culicoides pulicaris species complexes, implicated vectors of bluetongue virus in Europe. Vet Microbiol. 2007;124(1-2):82-94.

27. Schwenkenbecher JM, Mordueluntz AJ, Switek K, Piertney SB. Discrimination of Culicoides midge larvae using multiplex polymerase chain reaction assays based on DNA sequence variation at the mitochondrial cytochrome C oxidase I gene. J Med Entomol. 2009;46(3):610-4. 
28. Elbers ARW, Meiswinkel R, van Weezep E, van Oldruitenborgh-Oosterbaan MMS, Kooi EA. Schmallenberg Virus in Culicoides spp. Biting Midges, the Netherlands, 2011. Emerg Infect Dis. 2013;19(1):106-9.

29. Mellor HE, Hamilton JGC, Anderson M. Spectral sensitivity in the eyes of male and female Lutzomyia longipalpis sandflies. Med Vet Entomol. 1996:10(4):371-4.

30. Boorman J. British Culicoides (Diptera: Ceratopogonidae) notes on distribution and biology. Entomol Gaz. 1986;37(4):253-66.

31. Meiswinkel R, Scolamacchia F, Dik M, Mudde J, Dijkstra E, Van der Ven IJK, et al. The Mondrian matrix: Culicoides biting midge abundance and seasonal incidence during the 2006-2008 epidemic of bluetongue in the Netherlands. Med Vet Entomol. 2014;28(1):10-20.

32. Kettle DS, Lawson JWH. The early stage of the British biting midges Culicoides Latreille (Diptera, Ceratopogonidae) and allied genera. Bull Ent Res. 1952; 43(3):421-\&

33. Sanders CJ, Shortall CR, Gubbins S, Burgin L, Gloster J, Harrington R, et al Influence of season and meteorological parameters on flight activity of Culicoides biting midges. J Appl Ecol. 2011;48(6):1355-64.

34. Harrup LE, Logan JG, Cook Jl, Golding N, Birkett MA, Pickett JA, et al. Collection of Culicoides (Diptera: Ceratopogonidae) using $\mathrm{CO}_{2}$ and enantiomers of 1-octen-3-ol in the United Kingdom. J Med Entomol. 2012:49(1):112-21.

\section{Submit your next manuscript to BioMed Central and take full advantage of:}

- Convenient online submission

- Thorough peer review

- No space constraints or color figure charges

- Immediate publication on acceptance

- Inclusion in PubMed, CAS, Scopus and Google Scholar

- Research which is freely available for redistribution 